\title{
Variations of Light Curve Parameters in Miras with Progressive Period Changes
}

\author{
Vladislava I. Marsakova \\ Department of Astronomy, Odessa State University, \\ T. G. Shevchenko Park, Odessa 270014, Ukraine
}

\begin{abstract}
Variations of light curve parameters in Mira variables with progressive period changes have been studied. They lead to a consistent sequence of the stars which is related to the helium flash model.
\end{abstract}

There are four Mira stars with known progressive period changes: $\mathrm{R} \mathrm{Aql}$, R Hya, W Dra, T UMi. Wood \& Zarro (1981) and Gál \& Szatmáry (1995) discussed the period changes assuming the helium shell flash model.

We have used observations from AFOEV and VSOLJ databases to analyse period decreases and other light curve changes. The analysis is mainly analogous to the one described by Marsakova \& Andronov (1998).

In R Aql and R Hya the period changes are followed by strong amplitude changes as well as broadening of the light curve. In T UMi period changes are followed by sharpening of the light curve.

Using the changes of amplitude and the changes of slope of ascending and descending branches, we may construct a sequence for these stars. The relative rates of the change (multiplied by $10^{6}$ ) are listed below, where "-" corresponds to the case where the correlation with time is not higher than $3 \sigma$ :

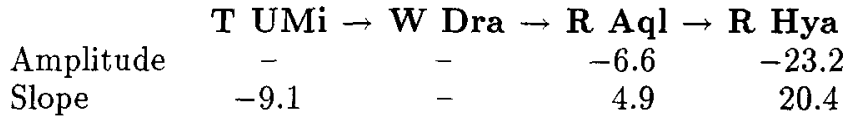

A consistent sequence of the four stars is revealed and it may be aligned with the increasing evolution of stars in the helium shell flash stage (Fig. 3 in Wood \& Zarro 1981), especially if we accept that T UMi is before the zero point of helium shell flash (Gál \& Szatmáry 1995).

Acknowledgments. Author is thankful to amateur astronomers from the AFOEV and VSOLJ for their observations and also to Prof. I. L. Andronov and Dr. P. R. Wood for helpful discussion and to the organizing committee and IAU for the travel grant.

\section{References}

Gál, J. \& Szatmáry, K. 1995, A\&A, 297, 461

Marsakova, V. I. \& Andronov, I. L. 1998, Ap\&SS, 257, 49

Wood, P. R. \& Zarro, D. M. 1981, ApJ, 247, 247 